\title{
Determining the best practicable control technology and its associated emission levels for Iron and Steel industry in Iran
}

\begin{abstract}
Industrial effluent limitations should be established regarding the special characteristics of each sector with emphasis given to the local context, whereas, in Iran, a uniform "Wastewater Effluent Standard" is employed throughout the country in which no specific industrial effluent limitations have been considered. This gap highlights the essential need for a transparent and scientifically proven methodology for determining effluent emission limit values (ELVs) at the sector level. In this research, an integrated approach is proposed based on the application of the Best Practicable Control Technology Currently Available (BPT) concept. The modelling framework includes a series of sequential steps comprising two main sections: 1. identifying the effluent emission datasets, and 2. computing the emission levels associated with the BPT (BPT-AELs). To identify the datasets, the methodology follows two different procedures: 1 . a detailed analysis of the environmental performance of this sector in the country to determine the plants reflecting BPT, and 2. screening their emission datasets through statistical analysis. The Best Available Techniques (BAT) principle is employed as a reference element for determining the best representative plants, and the results indicate that corrective measures in accordance with the BAT considerations should be implemented in many of the plants under consideration. The comparison of the derived BPT-AELs with the existing standards show that they are mainly lower than the standard levels and are reasonable estimations for the involved parameters. Conclusively, this methodology presents a reliable and practical stepwise process at the sector level, which can be developed for other industries.
\end{abstract}

Keyword: Iron and Steel industry; Effluent emission limit values; BPT-AELs; Censored data; Developing countries 